\title{
Hydrodynamic instabilities in the solar tachocline
}

\author{
F. Q. Rashid, C. A. Jones, and S. M. Tobias \\ University of Leeds, School Of Mathematics, Department of Applied Mathematics, University of Leeds, LS2 9JT Leeds, UK \\ e-mail: fayyaz@maths.leeds.ac.uk
}

Received 24 April 2008 / Accepted 27 June 2008

\begin{abstract}
Aims. We consider the stability of simple hydrodynamic models of the solar tachocline. This study addresses only the non-magnetic instabilities. In our model there is a strong radial shear, which because of the rotation is coupled to a latitudinal temperature gradient. We also assume there is a strong stable stratification, appropriate for the slow tachocline.

Methods. These instabilities are calculated by finding the eigenvalues and eigenfunctions using a matrix-based collocation method. We also find analytic expressions for the key instabilities in the relevant asymptotic limits of strong stable stratification and small Prandtl number.

Results. We find two distinct types of instability, axisymmetric modes of the Goldreich-Schubert type, and three-dimensional Eadytype modes. Both types of mode are affected by thermal diffusion. We find that the axisymmetric modes considered by Knobloch \& Spruit $(1982$, A\&A, 113, 261) are likely to dominate in the slow tachocline. The non-axisymmetric baroclinic instability of Eady-type may be important in the layers closer to the base of the convection zone.
\end{abstract}

Key words. hydrodynamics - instabilities - Sun: interior

\section{Introduction}

The solar tachocline is a layer of strong differential rotation at the base of the convection zone, (ChristensenDalsgaard \& Schou 1988; Spiegel \& Zahn 1992; Christensen-Dalsgaard \& Thompson 2007) which is believed to play an important role in the interior dynamics of the Sun. It is widely believed that the tachocline region has a strong influence in the generation of magnetic fields via dynamo action (Tobias \& Weiss 2007), and is responsible for coupling the radiative interior of the Sun to the convection zone (Gough 2007).

Helioseismology suggests that the tachocline is thin (Christensen-Dalsgaard \& Thompson 2007) of the order of $1 \%$ of the solar radius. It is of interest to understand why this shear layer is so thin (see e.g. Tobias 2005), and what processes can transport angular momentum in this region (Spiegel \& Zahn 1992; Gough \& McIntyre 1998). The tachocline is susceptible to a number of hydrodynamic and hydromagnetic instabilities (see e.g. Miesch 2005, for a review). These include magnetic buoyancy instabilities (Hughes 2007) and joint differential rotation and magnetic field instabilities (Gilman \& Cally 2007). Here we focus on the slow tachocline region where there is strong stable stratification with large Richardson number, where a more limited range of instabilities is possible.

Despite this strong stratification, GSF instabilities (Goldreich \& Schubert 1967; Fricke 1968) are possible, because the Prandtl number is small. This means the temperature gradient maintaining stability can be undermined by thermal diffusion if short wavelength disturbances are considered. Knobloch \& Spruit (1982) found that axisymmetric modes can become unstable provided the product of Richardson number and Prandtl number is less than a number of order unity.
The aim of this paper is to consider a simplified model of the solar tachocline, initially without the presence of a magnetic field, to study the instabilities that occur. In particular, we develop an asymptotic theory valid in the limit of small Ekman number, $E=v / 2 \Omega d^{2}$ where $v$ is kinematic viscosity and $d$ is the layer thickness, strongly stratified in the slow tachocline. Our model has the potential to be developed into the nonlinear regime, and a magnetic field will be added in future studies.

\section{The model}

We study the tachocline on a local scale (see Fig. 1). We consider a stably stratified layer lying between $z= \pm d / 2$ where $z$ represents depth, periodic in the eastward $x$-direction and the northward $y$-direction. Axisymmetric disturbances, which as we see below are the dominant instability in the strongly stratified regime, are independent of $x$. Since the tachocline layer is thin, we use the Boussinesq approximation. This thinness makes curvature effects small and for this reason we use Cartesian coordinates rather than spherical polar coordinates.

The equation of motion in a rotating frame with the Boussinesq approximation can be written as

$$
\frac{\partial \boldsymbol{u}}{\partial t}+(\boldsymbol{u} \cdot \nabla) \boldsymbol{u}+2 \boldsymbol{\Omega} \times \boldsymbol{u}=\frac{-\nabla p}{\rho}+g \alpha T \hat{z}+v \nabla^{2} \boldsymbol{u}
$$

where $\alpha$ is the thermal expansion coefficient and $T$ is the difference between the actual temperature and the adiabatic temperature of a spherically symmetric solar model. Moreover $\boldsymbol{\Omega}=$ $\Omega(0, \cos \lambda, \sin \lambda)=\left(0, \Omega_{y}, \Omega_{z}\right)$ is the rotation vector, and centrifugal acceleration is ignored in comparison with gravity. The temperature equation with thermal diffusivity $\kappa$ is

$$
\frac{\partial T}{\partial t}+(\boldsymbol{u} \cdot \nabla) T=\kappa \nabla^{2} T
$$




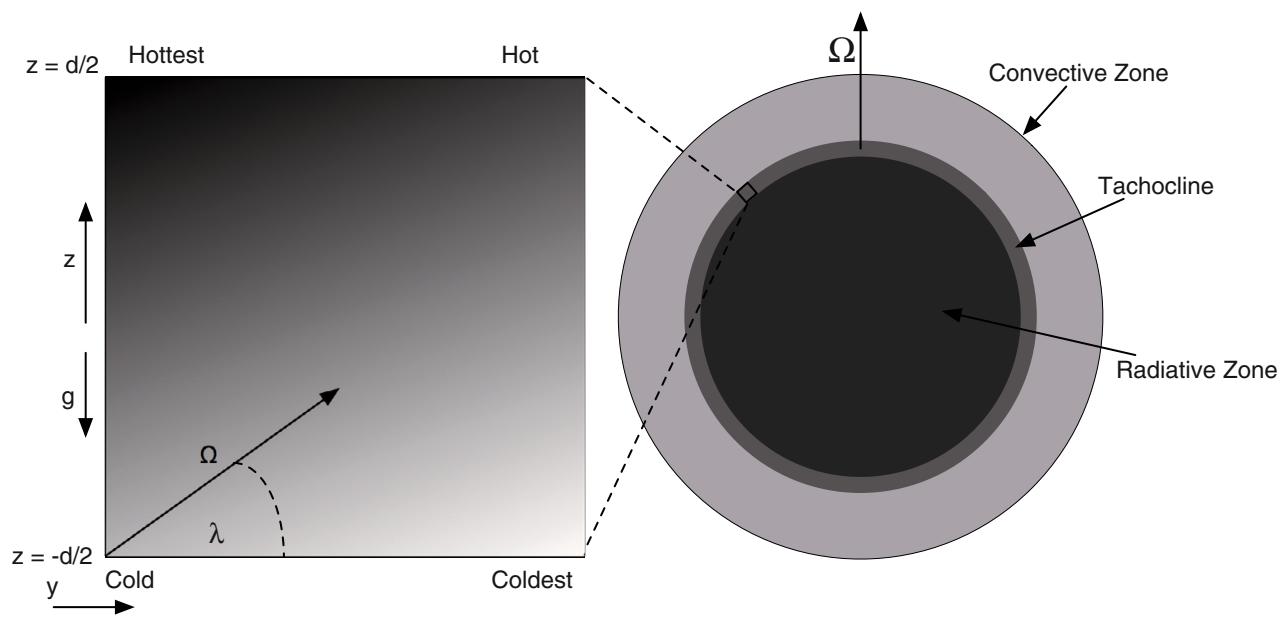

Fig. 1. We consider a periodic box in the $x$ (Eastward) and $y$ (Northward) directions. The thermal wind basic state flow is in the $x$-direction and varies with $z$, the radial direction. The temperatures marked are relative to the adiabatic temperature gradient, so there is subadiabatic stratification in $z$.

and the Boussinesq continuity equation is

$\nabla \cdot \boldsymbol{u}=0$.

\subsection{Basic state}

We assume that the flow is in the (zonal) $x$-direction and speed increases in the $z$-direction. We are therefore neglecting any latitudinal shear which in general is smaller in the tachocline, though there is a particular latitude region near $\pm 30^{\circ}$ where the radial shear is small and the latitudinal shear dominates. Here our assumption breaks down. We set

$\boldsymbol{u}_{0}=\left(U^{\prime} z, 0,0\right)$,

with $U^{\prime}$ assumed constant. Helioseismology suggests that $U^{\prime}>0$ for $|\lambda|<30^{\circ}$ and $U^{\prime}<0$ for $|\lambda|>30^{\circ}$ (ChristensenDalsgaaard \& Schou 1988). Taking the $x$-component of the curl of the equation of motion, this gives the basic state superadiabatic temperature as,

$T_{0}=\beta z-\frac{2 \Omega U^{\prime} \sin \lambda}{g \alpha} y$.

The basic state temperature therefore has a horizontal variation due to rotation, and a vertical variation due to stable stratification - for which $\beta>0$. This also explains why in Fig. 1 there is a gradient in the temperature in both $y$ and $z$ directions. Because we are ignoring any meridional shear, only the component of rotation in the radial direction, $\Omega \sin \lambda$, enters the thermal wind equation, so strong rotational effects will not apply to the region close to the equator, where $\sin \lambda$ vanishes.

\subsection{Perturbing the basic state}

We perform a linear stability analysis of the basic state by setting

$\boldsymbol{u}=\boldsymbol{u}_{0}+\boldsymbol{u}^{\prime}, \quad T=T_{0}+\theta, \quad p=p_{0}+p^{\prime}$,

$\boldsymbol{u}^{\prime}=\left(u_{x}, u_{y}, u_{z}\right)$. Here the perturbations are small in comparison with the basic state and linearising yields equations for the perturbations of the form

$\frac{\partial \boldsymbol{u}^{\prime}}{\partial t}+U^{\prime} z \frac{\partial \boldsymbol{u}^{\prime}}{\partial x}+U^{\prime} u_{z} \hat{\boldsymbol{x}}+2 \boldsymbol{\Omega} \times \boldsymbol{u}^{\prime}=-\nabla p^{\prime}+g \alpha \theta \hat{z}+v \nabla^{2} \boldsymbol{u}^{\prime}$, $\frac{\partial \theta}{\partial t}+U^{\prime} z \frac{\partial \theta}{\partial x}-\frac{2 \Omega_{z} U^{\prime}}{g \alpha} u_{y}+\beta u_{z}=\kappa \nabla^{2} \theta$

This gives four equations and five unknowns. The pressure is eliminated by taking the curl of (7) giving (with $\omega=\nabla \times \boldsymbol{u}^{\prime}$ )

$$
\begin{aligned}
& \frac{\partial \omega}{\partial t}+U^{\prime} z \frac{\partial \omega}{\partial x}+U^{\prime} \hat{z} \times \frac{\partial \boldsymbol{u}^{\prime}}{\partial x}+U^{\prime} \nabla \times u_{z} \hat{\boldsymbol{x}}-2(\boldsymbol{\Omega} . \nabla) \boldsymbol{u}^{\prime}= \\
& g \alpha \nabla \times \theta \hat{z}+v \nabla^{2} \omega .
\end{aligned}
$$

The $z$-component of (9) together with the $z$-component of the curl of (9) gives

$\frac{\partial \omega_{z}}{\partial t}+U^{\prime} z \frac{\partial \omega_{z}}{\partial x}-U^{\prime} \frac{\partial u_{z}}{\partial y}-2 \Omega_{y} \frac{\partial u_{z}}{\partial y}-2 \Omega_{z} \frac{\partial u_{z}}{\partial z}=v \nabla^{2} \omega_{z}$

$\frac{\partial \nabla^{2} u_{z}}{\partial t}+U^{\prime} z \frac{\partial \nabla^{2} u_{z}}{\partial x}+2 \Omega_{z} \frac{\partial \omega_{z}}{\partial z}+2 \Omega_{y} \frac{\partial \omega_{z}}{\partial y}=g \alpha \nabla_{\mathrm{H}}^{2} \theta+v \nabla^{4} u_{z}$

where $\nabla_{\mathrm{H}}^{2}=\partial^{2} / \partial x^{2}+\partial^{2} / \partial y^{2}$ is the horizontal Laplacian. Combining the continuity equation and the definition for $\omega_{z}$ yields

$\frac{\partial \omega_{z}}{\partial x}-\frac{\partial^{2} u_{z}}{\partial y \partial z}=\nabla_{\mathrm{H}}^{2} u_{y}$

This can be rearranged to eliminate $u_{y}$ in (8), so that

$$
\begin{array}{r}
\nabla_{\mathrm{H}}^{2}\left[\left(\frac{\partial}{\partial t}+U^{\prime} z \frac{\partial}{\partial x}\right) \theta+\beta u_{z}-\kappa \nabla^{2} \theta\right]= \\
\frac{2 \Omega_{z} U^{\prime}}{g \alpha}\left(\frac{\partial \omega_{z}}{\partial x}-\frac{\partial^{2} u_{z}}{\partial y \partial z}\right) .
\end{array}
$$

Equations (10), (11), and (13) give three equations with three unknowns. These are non-dimensionalised using the following relations:

$\frac{\partial}{\partial t}=2 \Omega \frac{\partial}{\partial \tilde{t}}, z=\tilde{z} d, u_{z}=U^{\prime} d \tilde{u}_{z}, \frac{\partial}{\partial x}=\frac{1}{d} \frac{\partial}{\partial \tilde{x}}$,

$\omega_{z}=U^{\prime} \tilde{\omega}_{z}, \quad \nabla^{2}=\frac{\tilde{\nabla}^{2}}{d^{2}}, \quad \theta=\frac{2 \Omega_{z} U^{\prime} d}{g \alpha} \tilde{\theta}$ 
This gives (the tilde having been dropped)

$\frac{\mathrm{D} \omega_{z}}{\mathrm{D} t}-R o \frac{\partial u_{z}}{\partial y}-\sin \lambda \frac{\partial u_{z}}{\partial z}-\cos \lambda \frac{\partial u_{z}}{\partial y}=E \nabla^{2} \omega_{z}$

$\frac{\mathrm{D}}{\mathrm{D} t} \nabla^{2} u_{z}+\sin \lambda \frac{\partial \omega_{z}}{\partial z}+\cos \lambda \frac{\partial \omega_{z}}{\partial y}=\nabla_{H}^{2} \theta+E \nabla^{4} u_{z}$

$\nabla_{\mathrm{H}}^{2}\left(\frac{\mathrm{D} \theta}{\mathrm{D} t}+R i R o^{2} u_{z}-E \operatorname{Pr}^{-1} \nabla^{2} \theta\right)=\sin \lambda R o\left(\frac{\partial \omega_{z}}{\partial x}-\frac{\partial^{2} u_{z}}{\partial y \partial z}\right)$

where $\quad \frac{\mathrm{D}}{\mathrm{D} t} \equiv \frac{\partial}{\partial t}+z R o \frac{\partial}{\partial x}$.

The dimensionless parameters are

$E=\frac{v}{2 \Omega d^{2}}, \quad R o=\frac{U^{\prime}}{2 \Omega}, \quad R i=\frac{g \alpha \beta}{\left(U^{\prime}\right)^{2}}, \quad \operatorname{Pr}=\frac{v}{\kappa}$,

where $E$ is the Ekman number, $R o$ is the Rossby number, $R i$ is the Richardson number and $P r$ is the Prandtl number.

The boundary conditions are taken as $u_{z}=0, \theta=0$, and in the stress-free case $\mathrm{d} \omega_{z} / \mathrm{d} z=0$ and $\mathrm{d}^{2} u_{z} / \mathrm{d} z^{2}=0$ on $z= \pm 1 / 2$. We concentrate on the stress-free case, but some numerical simulations were done with no-slip boundaries, with $\omega_{z}=0$ and $\mathrm{d} u_{z} / \mathrm{d} z=0$ on $z= \pm 1 / 2$.

\subsection{Axisymetric case}

We see below that axisymmetric modes, that is modes independent of $x$, are often dominant for low Prandtl numbers, and the equations can then be simplified. All $\partial / \partial x$ terms in (7) and (8) are ignored. We obtain

$\frac{\partial u_{x}}{\partial t}-v \nabla^{2} u_{x}-U^{\prime} \frac{\partial \psi}{\partial y}-2 \Omega_{z} \frac{\partial \psi}{\partial z}-2 \Omega_{y} \frac{\partial \psi}{\partial y}=0$,

$\frac{\partial \theta}{\partial t}-\kappa \nabla^{2} \theta-\frac{2 \Omega_{z} U^{\prime}}{g \alpha} \frac{\partial \psi}{\partial z}-\beta \frac{\partial \psi}{\partial y}=0$,

$\frac{\partial}{\partial t} \nabla^{2} \psi-v \nabla^{4} \psi+2 \Omega_{z} \frac{\partial u_{x}}{\partial z}+2 \Omega_{y} \frac{\partial u_{x}}{\partial y}=-g \alpha \frac{\partial \theta}{\partial y}$.

The function $\psi$ is a streamfunction with $u_{y}=\partial \psi / \partial z$ and $u_{z}=$ $-\partial \psi / \partial y$. The equations are non-dimensionalised using the previous relations including $\psi=U^{\prime} d^{2} \tilde{\psi}$ giving,

$\frac{\partial u_{x}}{\partial t}-E \nabla^{2} u_{x}-R o \frac{\partial \psi}{\partial y}-\sin \lambda \frac{\partial \psi}{\partial z}-\cos \lambda \frac{\partial \psi}{\partial y}=0$,

$\frac{\partial \theta}{\partial t}-\operatorname{Pr}^{-1} E \nabla^{2} \theta-\sin \lambda R o \frac{\partial \psi}{\partial z}-\operatorname{RiRo}^{2} \frac{\partial \psi}{\partial y}=0$,

$\frac{\partial}{\partial t} \nabla^{2} \psi-E \nabla^{4} \psi+\sin \lambda \frac{\partial u_{x}}{\partial z}+\cos \lambda \frac{\partial u_{x}}{\partial y}+\frac{\partial \theta}{\partial y}=0$,

the tildes being dropped and the dimensionless parameters defined as before. Stress-free boundary conditions are now given by $\psi=\theta=\mathrm{d} u_{x} / \mathrm{d} x=\mathrm{d}^{2} \psi / \mathrm{d} z^{2}=0$ on $z= \pm 1 / 2$.

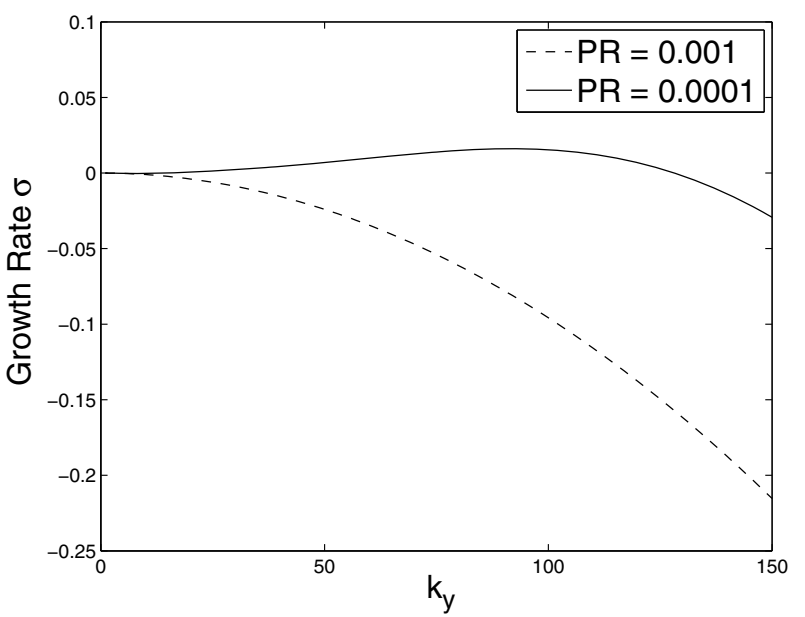

Fig. 2. The growth-rate of the axisymmetric mode as a function of wavenumber $k_{y}$ at $P r=10^{-3}$ and $P r=10^{-4}$ for $R i=2000, R o=1$, $E=10^{-5}, \lambda=90^{\circ}$. The axisymmetric mode is stable for all wavenumbers at $\operatorname{Pr}=10^{-3}$, but growing modes occur for $\operatorname{Pr}=10^{-4}$, with fastest growth-rate at $k_{y} \approx 92$.

\section{Numerics}

We solved the non-axisymmetric and axisymmetric equations using spectral methods. We seek solutions of the form $\sim \exp \left(\sigma t+\mathrm{i} k_{x} x+\mathrm{i} k_{y} y\right)$, where the (possibly complex) growth rate is $\sigma$, and $k_{x}$ and $k_{y}$ are the wavenumbers in the azimuthal $x$ direction and the latitudinal $y$ direction respectively. The model has seven parameters $\left(R o, E, \lambda, k_{x}, k_{y}, P r, R i\right)$ that can be varied to find the growth rate $\sigma$. To reduce the parameter space we concentrate on the typical cases with $R o=1$ and $\lambda=90^{\circ}$. The eigenvalue code was checked against the numerical results of Miller \& Antar (1986) and successfully reproduced their Fig. 2 results.

\subsection{Preference of axisymmetric modes at low Prandtl number}

We commenced by using the non-axisymmetric equations to show that axisymmetric modes $\left(k_{x}=0\right)$ are generally preferred over 3D $\left(k_{x}, k_{y} \neq 0\right)$ modes at low Prandtl number. Here the Ekman number was set at $E=10^{-5}$, and stress-free boundaries were used for these experiments. The Prandtl number was varied to find when different modes become unstable. In Fig. 2, the axisymmetric equations are solved for $R i=2000$. When $P r=0.001$, all axisymmetric modes are stable for all wavenumbers; the least damped mode is shown in Fig. 2. This is expected due to the strong stabilising stratification. However, at $P r=0.0001$ there is a range of wavenumbers for which there is a growing mode. At small Prandtl number the effect of thermal diffusion weakens the effect of the stratification, particularly for short wavelength modes. Note that the fastest growing mode has the large wavenumber $k_{y} \approx 92$, so these are fingering modes, as expected from the analysis of Knobloch \& Spruit (1982).

In Fig. 3, the 3D code solving Eqs. (15)-(17) with stressfree boundaries and $R i=2000$ and $\operatorname{Pr}=10^{-4}$ was used to plot the growth rate as a function of the wavenumbers $k_{x}$ and $k_{y}$. In Fig. 3a, the maximum growth rate is seen to be at $k_{y}=92$ as before, and we see that non-zero $k_{x}$ weakens this essentially axisymmetric instability. The neutral line is shown to enclose a region of instability centred on $k_{y}=92$ and $k_{x}=0$. However, if the small wavenumber range is examined in detail, as in Fig. 3b, 
(a)

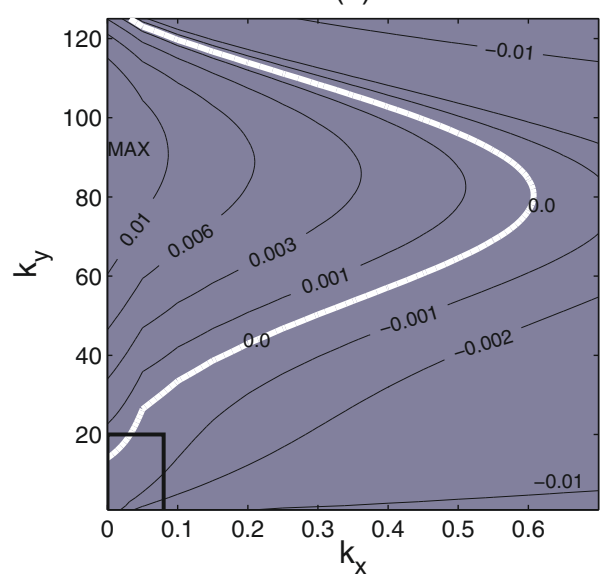

(c)

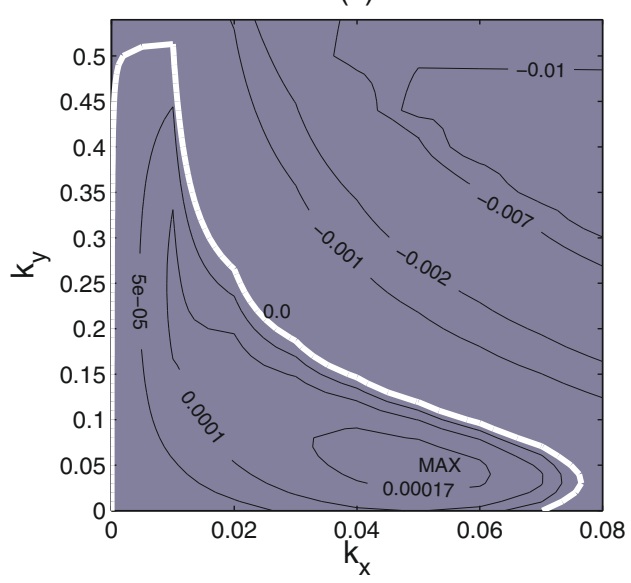

(b)

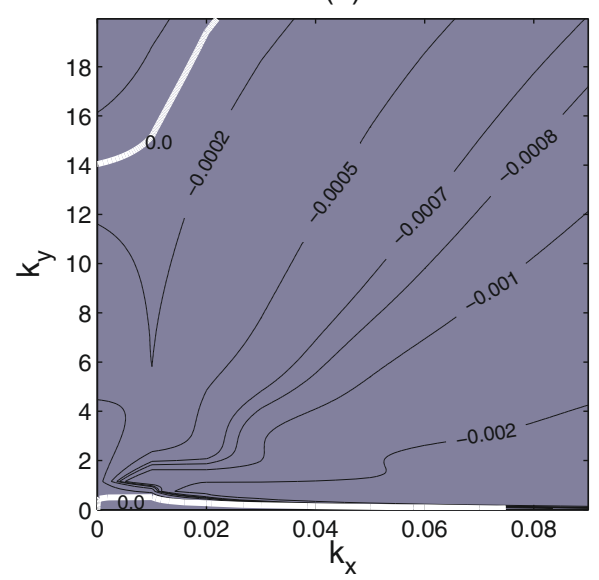

(d)

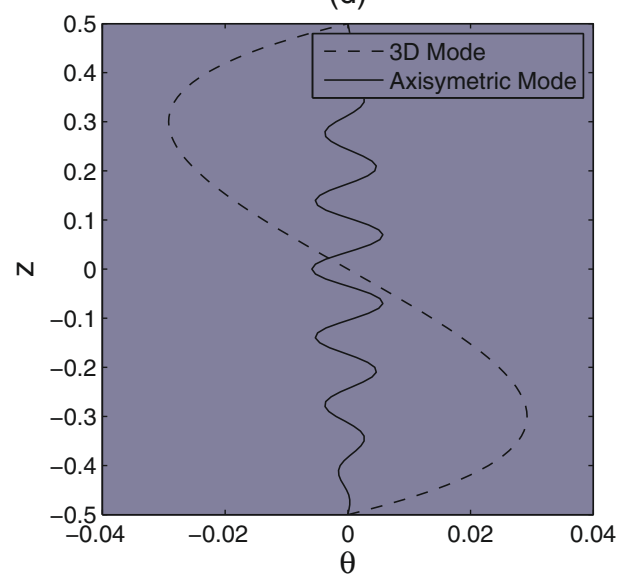

Fig. 3. The contour plots show the growth rate $\sigma$ plotted against $k_{x}$ and $k_{y}$, the zero growth-rate contours are shown in heavy white. a) is showing that the axisymmetric modes dominate at low Prandtl number. The maximum occurs at $k_{y}=92$ and $k_{x}=0$ with a growth-rate of $\sigma=0.01604$. b) is a close-up of the bottom left hand box in a). Two zero-growth contours appear. c) is a close-up of the bottom zero growth-rate contour in b), showing the appearance of non-axisymmetric instability at very low $k_{x}$ and $k_{y}$. The greatest of these instabilities occurs at $k_{y}=k_{x}=0.05$ with a growth-rate of $\sigma=0.000206$. d) shows the eigenfuctions (real part of $\theta$ is plotted) of the two local maximum growth-rate points. These were plotted at $R i=2000, \operatorname{Pr}=10^{-4}, E=10^{-5}, \operatorname{Ro}=1$ and $\lambda=90^{\circ}$.

we see that a second neutral curve appears with low $k_{x}$ and $k_{y}$. In Fig. 3c, we blow this region up even more, and we see there is an unstable growing non-axisymmetric mode with maximum growth at $k_{x}=k_{y}=0.05$. This growing non-axisymmetric mode occurs over a wide range of Prandtl numbers, but it has a low growth rate. In Fig. 3 the maximum growth rate for the axisymmetric instability is 0.01604 , whereas the maximum growth rate for the low wavenumber non-axisymmetric instability is only 0.000206 . So when the axisymmetric instability occurs, it very soon dominates the non-axisymmetric mode. In Fig. 3d the real part of the temperature perturbation is shown for the two types of mode at their maximum growth rate. The axisymmetric mode has many nodes, showing that the fingers slope in the $y-z$ plane, while the non-axisymmetric mode is larger scale and very smooth. For the relevant small $E$ and $P r$ and large $R i$ limit we see below that the asymptotic structure of both types of mode can be found analytically.

These findings are summarised in Table 1, which contains data for various values of the Richardson number. We denote the Prandtl number at which axisymmetric instability first occurs as $\operatorname{Pr}$ is reduced by $\operatorname{Pr}_{A x}$. As $\operatorname{Pr}$ is further reduced,
Table 1. Table showing when axisymmetric modes become unstable $\left(P r_{A x}\right)$ and when their growth rate is the same as the growth rate of the 3D modes $\left(P r_{C x}\right)$, for different Richardson numbers. The axisymmetric modes became unstable at wavenumber about $k_{y} \approx 54$ for this value of $E=10^{-5}$, Ro $=1$ and $\lambda=90^{\circ}$.

\begin{tabular}{ccccc}
\hline \hline$R i$ & $\operatorname{Pr}_{A x}\left(10^{-3}\right)$ & $\operatorname{Pr}_{C x}\left(10^{-3}\right)$ & $\operatorname{RiPr}_{A x}$ & $\operatorname{RiPr}_{C x}$ \\
\hline 200 & 1.2280 & 1.0702 & 0.2455 & 0.2140 \\
500 & 0.4904 & 0.4710 & 0.2452 & 0.2355 \\
750 & 0.3268 & 0.3198 & 0.2451 & 0.2398 \\
1000 & 0.2451 & 0.2417 & 0.2451 & 0.2417 \\
1500 & 0.1633 & 0.1621 & 0.2450 & 0.2431 \\
1750 & 0.1400 & 0.1391 & 0.2450 & 0.2434 \\
2000 & 0.1228 & 0.1218 & 0.2450 & 0.2437 \\
\hline
\end{tabular}

the axisymmetric growth rate grows rapidly while the nonaxisymmetric growth rate does not, so at $\operatorname{Pr}_{C x}$ the cross-over point is reached where the axisymmetric mode grows faster than the non-axisymmetric mode, so at $P r<\operatorname{Pr}_{C x}$ axisymmetric instability is preferred. The asymptotic theory described below 
Table 2. Numerically computed values of $\operatorname{RiPr}$ and $k_{y}$ at the onset of instability using stress-free boundary conditions. $\operatorname{Ro}=1, \lambda=90^{\circ}$.

\begin{tabular}{ccccccccc}
\hline \hline & $E=10^{-3}$ & \multicolumn{3}{c}{$E=10^{-4}$} & \multicolumn{3}{c}{$E=10^{-5}$} & \multicolumn{3}{c}{$E=10^{-6}$} \\
$\operatorname{Pr}$ & $\operatorname{RiPr}$ & $k_{y}$ & $\operatorname{RiPr}$ & $k_{y}$ & $\operatorname{RiPr}$ & $k_{y}$ & $\operatorname{RiPr}$ & $k_{y}$ \\
\hline 0.100000 & 0.17932 & 11.42280 & 0.27760 & 24.60042 & 0.29722 & 53.04886 & 0.30137 & 114.32014 \\
0.010000 & 0.13670 & 11.61718 & 0.23106 & 25.05555 & 0.24994 & 54.04072 & 0.25393 & 116.46144 \\
0.001000 & 0.13264 & 11.63590 & 0.22662 & 25.10044 & 0.24543 & 54.13850 & 0.24941 & 116.67173 \\
0.000100 & 0.13223 & 11.63536 & 0.22618 & 25.10492 & 0.24498 & 54.15090 & 0.24896 & 116.69190 \\
0.000010 & 0.13219 & 11.63427 & 0.22614 & 25.12161 & 0.24494 & 54.12948 & 0.24892 & 116.68706 \\
0.000001 & 0.13225 & 11.59217 & 0.22613 & 25.11130 & 0.24493 & 54.13075 & 0.24891 & 116.68805 \\
\hline
\end{tabular}

suggests that $\operatorname{RiPr}$ must be less than $1 / 4$ for instability at small $E$ and large $R i$, and so we show $\operatorname{RiPr}_{A x}$ and $\operatorname{RiPr}_{c x}$ in Table 1.

\subsection{Axisymmetric modes and the Ekman number}

Using the axisymmetric Eqs. (19)-(21), we found there is a relationship between the Ekman number and the wavenumber $k_{y}$ for when the axisymmetric mode becomes unstable. As $R i$ is reduced there is a critical $k_{y}$ at which instability first onsets. In Table 2 we give the critical values of $R i P r$ for onset on instability, together with the corresponding $k_{y}$, for a range of $E$ and $P r$. We see that in the tachocline regime, onset always occurs when $\operatorname{RiPr} \approx 0.25$. The value of $k_{y}$ at critical increases as $E$ is reduced. The table here is for stress-free boundaries. The case of no-slip boundaries was also examined, but there is no great difference betwen the no-slip and stress-free cases.

\section{Local dispersion relation}

The oscillatory nature of the axisymmetric eigenfunction, Fig. 3d, suggests that a local stability analysis assuming perturbations are periodic in $y$ and $z, \sim \exp \left(\sigma t+\mathrm{i} k_{y} y+\mathrm{i} k_{z} z\right)$ is informative. Substituting this into Eqs. (19)-(21) gives (note $k^{2}=k_{y}^{2}+k_{z}^{2}$ )

$\sigma u_{x}+E k^{2} u_{x}-\mathrm{i} k_{y} R o \psi-\mathrm{i} k_{z} \psi=0$,

$\sigma \theta+\operatorname{Pr}^{-1} E k^{2} \theta-\mathrm{i} k_{z} R o \psi-\mathrm{i} k_{y} R i R o^{2} \psi=0$,

$-\sigma k^{2} \psi-E k^{4} \psi+\mathrm{i} k_{z} u_{x}+\mathrm{i} k_{y} \theta=0$.

Eliminating the variables $\psi, \theta, u_{x}$, gives the local dispersion relation

$$
\begin{aligned}
& \sigma^{3}+\sigma^{2} E k^{2}\left(\operatorname{Pr}^{-1}+2\right)+\sigma\left[E^{2} k^{4}\left(1+2 \operatorname{Pr}^{-1}\right)\right. \\
& \left.+\frac{2 k_{y} k_{z} R o}{k^{2}}+\frac{k_{z}^{2} R o}{k^{2}}+\frac{k_{y}^{2} R i R o}{k^{2}}\right]+E\left[E^{2} k^{6} \operatorname{Pr}^{-1}\right. \\
& \left.+k_{y} k_{z} \operatorname{Ro}\left(1+\operatorname{Pr}^{-1}\right)+\operatorname{Pr}^{-1} k_{z}^{2}+\operatorname{RiRo}^{2} k_{y}^{2}\right]=0 .
\end{aligned}
$$

\subsection{Diffusionless case $(v=\kappa=0)$}

In the diffusionless limit $E \rightarrow 0$ with $\operatorname{Pr}$ finite, The local dispersion relation becomes

$\sigma^{3}+\sigma\left[\frac{2 k_{y} k_{z} R o}{k^{2}}+\frac{k_{z}^{2} R o}{k^{2}}+\frac{k_{y}^{2} R i R o}{k^{2}}\right]=0$.
Noting there is also a solution with $\sigma=0$, we see this gives wave-like solutions. We can write

$$
-\sigma^{2}=\left(\frac{k_{z}}{k}+\frac{k_{y} R o}{k}\right)^{2}+\frac{k_{y}^{2} R o^{2}}{k^{2}}(R i-1) .
$$

This provides some physical insight into the nature of instability in these baroclinic systems. If there were no stable stratification, i.e. for $R i=0$, Eq. (30) reduces to

$$
-\sigma^{2}=\frac{k_{z}^{2}}{k^{2}}\left(1+\frac{2 k_{y} R o}{k_{z}}\right) .
$$

This gives growing baroclinic instability modes if $1+2 R_{0} k_{y} / k_{z}<0$. When the unstable wavevectors are plotted in the $k_{y}-k_{z}$ plane they have arguments $\phi$ which lie in the wedge $-\tan ^{-1} 2 R o<\phi<0$. Since $\boldsymbol{u}=\boldsymbol{u}_{0} \exp \mathrm{i}\left(k_{y} y+k_{z} z\right)$, and $\nabla \cdot \boldsymbol{u}=0$, it follows that $\boldsymbol{k} \cdot \boldsymbol{u}_{0}=0$, so that the flow in the $y-z$ plane is perpendicular to the wavevector. Hence unstable modes are those where the flow in the $y-z$ plane is in the wedge $\pi / 2-\tan ^{-1} 2 R o<\phi<\pi / 2$. Particles of fluid moving in this wedge are accelerated in the $x$-direction in such a way that this $x$-flow enhances the motion along the wedge. Note that as the thermal wind becomes small, i.e. for $R o \rightarrow 0$, the wedge angle of instability closes up to a narrow angle.

However, provided that $R i>1$, Eq. (30) shows that $\sigma^{2}$ is always negative so setting $\sigma=-\mathrm{i} \omega$ the dispersion relation is

$\omega=\frac{1}{k} \sqrt{\left(k_{z}+k_{y} R o\right)^{2}+k_{y}^{2} R o^{2}(R i-1)}$.

In the tachocline the Richardson number is believed to be large, so in this case we have wave-like stable solutions, corresponding to inertial-gravity waves. In the limit $k_{z} \rightarrow 0$ we have pure internal gravity waves, since $R i>0$ implies stable stratification, whereas in the limit $k_{y} \rightarrow 0$ we have inertial waves with frequency $2 \Omega$ in dimensional units. The slightly unusual form of this inertial-gravity wave dispersion relation is a consequence of the baroclinic sloping contours of constant temperature, see Fig. 1.

\subsection{Asymptotically small Ekman number}

We now assume $0<E \ll 1$. The inviscid modes that gave the inertial-gravity waves are now damped because of viscosity (see below). We therefore focus on the mode that had $\sigma=0$ in the case $E=0$, which now becomes significant. It has $\sigma \sim O(E)$ reducing the dispersion relation to first order,

$\sigma=-E k^{2}\left(1+\frac{\left(P r^{-1}-1\right)\left(k_{y} R o+k_{z}\right) k_{z}}{\left(k_{z}+k_{y} R o\right)^{2}+k_{y}^{2} R o^{2}(R i-1)}\right)$. 
If $\operatorname{Pr}=1$ then $\sigma=-E k^{2}$ which gives stable modes. However in the tachocline $\operatorname{Pr} \ll 1$ and this allows unstable modes even though $R i \gg 1$. Since $R i-1>0$, we can multiply through by the positive denominator, to obtain the condition for stability, $\sigma<0$,

$\left(k_{z}+\frac{1}{2} k_{y} R o\right)^{2}+k_{y}^{2} \operatorname{Ro}^{2}\left(\operatorname{PrRi}-\frac{1}{4}\right)+\operatorname{PrRok}_{y} k_{z}>0$.

The Prandtl number in the solar tachocline is estimated at $2 \times 10^{-6}$, while $R o$ is of order unity or a little less, so we can neglect the $\operatorname{PrRok}_{y} k_{z}$ term. We cannot neglect the $\operatorname{PrR} i$ term because $R i$ is very large. Clearly we have stability if $\operatorname{RiPr}>1 / 4$, but if $\operatorname{RiPr}<1 / 4$ we expect instability to occur, because wavenumbers can be selected so that

$k_{z}+\frac{1}{2} k_{y} R o=0$.

Note that these steady growing modes have their wavevectors pointing in the wedge of instability determined by (31). Physically, the large thermal diffusion coming from low $\operatorname{Pr}$ has weakened the stabilising stratification, thus allowing the baroclinic instability mechanism to operate again. The mode $\sigma=$ $\pm \mathrm{i} \omega$ when $E=0$ becomes stable as $E$ increases at low $\operatorname{Pr}$ and large $R i$, so the inertial-gravity waves are damped by small diffusion.

In dimensional units, the growth rate is

$\sigma=\kappa k^{2}\left[\frac{k_{y}^{2} U^{\prime 2}\left(\frac{1}{4}-\frac{g \alpha \beta v}{\kappa U^{\prime 2}}-\frac{2 \Omega k_{z} v}{\kappa U^{\prime} k_{y}}\right)-\left(2 \Omega k_{z}+\frac{k_{y} U^{\prime}}{2}\right)^{2}}{4 \Omega^{2} k_{z}^{2}+4 k_{y} k_{z} \Omega U^{\prime}+k_{y}^{2} g \alpha \beta}\right]$.

Selecting the wavenumbers $k_{y}$ and $k_{z}$ so that $2 \Omega k_{z}+k_{y} U^{\prime} / 2=0$, taking the limit of small viscosity, so $\operatorname{RiPr}=g \alpha \beta v / \kappa U^{\prime 2} \ll 1 / 4$, and large $R i$ so $4 \Omega^{2} k_{z}^{2}+4 k_{y} k_{z} \Omega U^{\prime} \ll k_{y}^{2} g \alpha \beta$, we obtain

$\sigma \sim \kappa k^{2} R i^{-1}=\frac{\kappa k^{2} U^{\prime 2}}{4 g \alpha \beta}$.

This is the thermal diffusion time for modes of wavenumber $k$ divided by four times the Richardson number, which measures the strength of the stable stratification. We see below that $k$ can be large, so the thermal time can be short. Indeed, the magnitude of the permissible $k$ values is only limited by the very small viscous diffusion. So we expect fingering type modes to grow rapidly even if the Richardson number is large.

\section{Asymptotics}

\subsection{Axisymmetric equations}

For axisymmetric modes we may determine the values of $k_{y}$ and $\operatorname{PrR} i$ that give steady modes using asymptotic methods. We consider the limit $E \rightarrow 0, R i \rightarrow \infty$ and $\operatorname{Pr} \rightarrow 0$ with $\operatorname{RiPr}$ and $\operatorname{Ro}$ remaining finite. For simplicity, we assume $\lambda=90^{\circ}$ and look for neutral modes with $\sigma=0$. The non-dimensional linearised equations for small disturbances to the basic state (22) - (24) become

$$
\begin{aligned}
& E \nabla^{2} u_{x}+R o \frac{\partial \psi}{\partial y}+\frac{\partial \psi}{\partial z}=0, \\
& E \nabla^{2} \theta+S R^{2} \frac{\partial \psi}{\partial y}=0, \\
& -E \nabla^{4} \psi+\frac{\partial u_{x}}{\partial z}+\frac{\partial \theta}{\partial y}=0,
\end{aligned}
$$

where the term $\operatorname{PrRo} \partial \psi / \partial z$ in (23) can be neglected because $P r$ is small, and we let $S=P r R i$, since $P r$ and $R i$ only appear in this combination. We apply $E \nabla^{2}$ to Eq. (40), then rearrange the equations to find a single PDE in $\psi$,

$E^{2} \nabla^{6} \psi+\frac{\partial^{2} \psi}{\partial z^{2}}+R o \frac{\partial^{2} \psi}{\partial y \partial z}+S R o^{2} \frac{\partial^{2} \psi}{\partial y^{2}}=0$

We seek solutions of the form $\psi(y, z)=\tilde{\psi}(z) \exp \left(\mathrm{i} k_{y} y\right)$, therefore

$E^{2}\left(\mathrm{D}^{2}-k_{y}^{2}\right)^{3} \psi+\mathrm{D}^{2} \psi+\mathrm{i} k_{y} R o \mathrm{D} \psi-k_{y}^{2} S R o^{2} \psi=0$.

The tilde has been dropped and the operator $D$ represents differentiation with respect to $z$. The equation above is a 6th order differential equation, requiring 6 boundary conditions to solve it. Either no-slip or stress-free boundary conditions can be used.

We look for solutions of the form

$\psi(z)=\sum_{i=1}^{i=6} A_{i} \exp \left(m_{i} z\right)$

where the $m_{i}$ are solutions of

$E^{2}\left(m^{2}-k_{y}^{2}\right)^{3}+m^{2}+\mathrm{i} k_{y} m R o-k_{y}^{2} S R o^{2}=0$.

Equation (44) has two roots in the limit $E \rightarrow 0$ which give the interior part of the solution, and another four roots with $m \sim$ $E^{-1 / 2}$ which determine the Ekman boundary layer structure. We start by setting $E=0$,

$m^{2}+\mathrm{i} k_{y} m R o-k_{y}^{2} S R o^{2}=0$,

giving

$m=\frac{-\mathrm{i} k_{y} R o}{2} \pm \frac{k_{y} R o}{4} \sqrt{S-\frac{1}{4}}$.

From Sect. 4.2 we know that for critical modes $S \rightarrow 1 / 4$, therefore we have a double root at leading order

$m=\frac{-\mathrm{i} k_{y} R o}{2}$

Note also that $m$ then has the expected form from (35), since $m=$ $\mathrm{i} k_{z}$. This leading order solution does not satisfy the boundary conditions $\psi=0$ on $z= \pm 1 / 2$, so $k_{y}$ and $k_{z}$ must be large, and $\psi$ is modulated on an order one length scale. At next order $m=$ $\left(-\mathrm{i} k_{y} R o\right) / 2+O(1)$. This implies that $\left(k_{y} R o / 4\right) \sqrt{S-1 / 4} \sim O(1)$. We set $S=1 / 4-\widetilde{S}$ so that $\left(\mathrm{i} k_{y} R o\right) / 4 \sqrt{\widetilde{S}} \sim O(1)$. To satisfy the boundary conditions we need (46) to be of the form

$m_{ \pm}=-\left(\mathrm{i} k_{y} R o\right) / 2 \pm \mathrm{i} n \pi$

for some integer $n$. This gives

$\left(k_{y} R o / 4\right) \sqrt{1 / 4-S}=n \pi$.

This predicts that $n=1$ is the first mode to become unstable as $S$ is reduced below $1 / 4$, so

$\psi(z)=A \exp \left(-\frac{\mathrm{i} k_{y} R o}{2} z\right) \cos (\pi z)$

which correctly satisfies the boundary conditions $\psi=0$ on $z=$ $\pm 1 / 2$, but it also predicts that instability can occur arbitrarily close to $1 / 4$ provided $k_{y}$ is very large. However at very large $k_{y}$, the term $E^{2}\left(m^{2}-k_{y}^{2}\right)^{3}$ in (44) becomes important, and it is this term which sets the limit on how large $k_{y}$ can be. If we assume 
Table 3. Calculated values for $k_{y}$ and $S_{\mathrm{c}}$ when $R o=1$ using Eqs. (53) and (55).

\begin{tabular}{ccc}
\hline \hline$E$ & $k_{y}$ & $S_{\mathrm{c}}$ \\
\hline $10^{-3}$ & 11.67051314 & 0.141304616 \\
$10^{-4}$ & 25.14335836 & 0.226582289 \\
$10^{-5}$ & 54.16972348 & 0.244954807 \\
$10^{-6}$ & 116.7051314 & 0.248913046 \\
\hline
\end{tabular}

$k_{y} \sim E^{-\alpha}$ for some fixed $\alpha$, then $\widetilde{S} \sim E^{2 \alpha}$. Also to determine the critical $k_{y}$, we need $E^{2}\left(m^{2}-k_{y}^{2}\right)^{3} \sim O(1)$, i.e. $E^{2} E^{-6 \alpha} \sim O(1) \Rightarrow$ $\alpha=1 / 3$.

Substituting the $m$ given by (48) with $n=1$ into (44) and equating $O(1)$ terms,

$\hat{S}=E^{-\frac{2}{3}} \frac{\pi^{2}}{k_{y}^{2} R o^{2}}+\frac{E^{\frac{4}{3}} k_{y}^{4}}{R o^{2}}\left(1+\frac{R o^{2}}{4}\right)^{3}$

where $\widetilde{S}=\hat{S} E^{\frac{2}{3}}$. Minimising $\hat{S}$ over $k_{y}^{2}$,

$\frac{\mathrm{d} \hat{S}}{\mathrm{~d} k_{y}^{2}}=-E^{-\frac{2}{3}} \frac{\pi^{2}}{k_{y}^{4} R o^{2}}+\frac{2 k_{y}^{2} E^{\frac{4}{3}}}{R o^{2}}\left(1+\frac{R o^{2}}{4}\right)^{3}=0$,

giving

$k_{y}=\frac{\pi^{\frac{1}{3}} E^{-\frac{1}{3}}}{2^{\frac{1}{6}}}\left(1+\frac{R o^{2}}{4}\right)^{-\frac{1}{2}}$.

The value of $\hat{S}$ at this minimising critical $k_{y}$ is

$\hat{S}=\frac{3 \pi^{\frac{4}{3}}}{2^{\frac{2}{3}} R o^{2}}\left(1+\frac{R o^{2}}{4}\right)$

so the critical value of $S$,

$S_{\mathrm{c}}=\frac{1}{4}-\frac{3 \pi^{\frac{4}{3}}}{2^{\frac{2}{3}}}\left(\frac{1}{R o^{2}}+\frac{1}{4}\right) E^{\frac{2}{3}}$.

In Table 3 we show some values of $k_{y}$ and $S_{\mathrm{c}}$ using these asymptotic formulae (53) and (55). These compare very satisfactorily with the equivalent values in Table 2 , obtained by solving the full problem numerically.

To compare with the results in Table 1, we need an asymptotic formula for the growth rate. Restoring the terms $-\sigma u_{x}$, $-\sigma \operatorname{Pr} \theta$ and $\sigma \nabla^{2} \psi$ to (38), (39) and (40) respectively, and taking the limit $\mathrm{Pr} \rightarrow 0$, we obtain at leading order

$\sigma S R o^{2} \frac{\partial^{2} \psi}{\partial y^{2}}=E^{3} \nabla^{8} \psi+E \nabla^{2}\left(\frac{\partial^{2} \psi}{\partial z^{2}}+R o \frac{\partial^{2} \psi}{\partial y \partial z}+S R o^{2} \frac{\partial^{2} \psi}{\partial y^{2}}\right)$,

giving

$\sigma=E\left(1+\frac{4}{R o^{2}}\right)\left[E^{2 / 3} \hat{S} R o^{2} k_{y}^{2}-\pi^{2}-E^{2} k_{y}^{6}\left(1+\frac{R o^{2}}{4}\right)^{3}\right]$.

Maximising this over $k_{y}$, we get the growth rate for axisymmetric modes,

$\sigma_{a x}=E\left(1+\frac{4}{R o^{2}}\right)\left[\frac{2 \hat{S}^{3 / 2} R o^{3}}{3^{3 / 2}\left(1+R o^{2} / 4\right)^{3 / 2}}-\pi^{2}\right]$.
Finally, we note that the problem at general latitude $\lambda$ is solved using the same analysis. The generalisation of (53) and (55) is

$k_{y}=\frac{\pi^{\frac{1}{3}} E^{-\frac{1}{3}} \sin ^{\frac{4}{3}} \lambda}{2^{\frac{1}{6}}}\left(1+\frac{R o^{2}}{4}+\cos \lambda R o\right)^{-\frac{1}{2}}$,

$S_{\mathrm{c}}=\frac{1}{4}-\frac{3 \pi^{\frac{4}{3}}}{2^{\frac{2}{3}}}\left(\frac{1}{R o^{2}}+\frac{\cos \lambda}{R o}+\frac{1}{4}\right) E^{\frac{2}{3}}$.

The general behaviour of cases where $R o \sim 1$ and $\lambda$ is in the midlatitudes is very similar to that of the case $R o=1$ and $\lambda=90^{\circ}$.

\subsection{Non-axisymmetric equations}

The low values of $k_{x}$ and $k_{y}$ found in the numerical results for the 3D modes, see Fig. 3c, suggest that asymptotic analysis is possible for the unstable 3D modes also - although a different balance is necessary for these modes. As before, we take the limits $E \rightarrow 0, R i \rightarrow \infty$ and $P r \rightarrow 0$ with $R i P r$ and $R o$ remaining finite. Here we also link $E$ and $R i$ so that $E R i=\hat{E}$ remains finite. For simplicity we set $\lambda=90^{\circ}$ and $R o=1$, though the same method works for general $R o$ and $\lambda$.

We assume disturbances $\sim \exp \left(\sigma t+\mathrm{i} k_{x} x+\mathrm{i} k_{y} y\right)$ and let $\epsilon=$ $R i^{-1 / 2}$. We also set

$k_{x}=\epsilon \hat{k}_{x}, \quad k_{y}=\epsilon \hat{k}_{y}, \quad \sigma=\epsilon^{2} \hat{\sigma}$.

Since $\operatorname{RiPr}=S$, we have $\operatorname{Pr}=S \epsilon^{2}$ and $E=\hat{E} \epsilon^{2}$. We use Eqs. (15)-(17) with the ansatz

$\omega_{z}=\epsilon \hat{\omega}_{1}+\epsilon^{2} \hat{\omega}_{2}+\epsilon^{3} \hat{\omega}_{3}+\cdots$,

$u_{z}=\epsilon^{2} \hat{u}_{2}+\epsilon^{3} \hat{u}_{3}+\epsilon^{4} \hat{u}_{4}+\cdots$,

$\theta=\hat{\theta}_{0}+\epsilon \hat{\theta}_{1}+\epsilon^{2} \hat{\theta}_{2}+\cdots$

equating similar powers of $\epsilon$ at each order. At leading order, which is $O\left(\epsilon^{2}\right)$ for (15), $O(\epsilon)$ for (16) and (17), we obtain

$\mathrm{i} k_{x} z \hat{\omega}_{1}=\frac{\mathrm{d} \hat{u}_{2}}{\mathrm{~d} z}$

$\frac{\mathrm{d} \hat{\omega}_{1}}{\mathrm{~d} z}=0$

$-\hat{a}^{2} \hat{u}_{2}+\frac{\hat{E} \hat{a}^{2}}{S} \frac{\mathrm{d}^{2} \hat{\theta}_{0}}{\mathrm{~d} z^{2}}=\mathrm{i} \hat{k}_{x} \hat{\omega}_{1}, \hat{a}^{2}=\hat{k}_{x}^{2}+\hat{k}_{y}^{2}$

(66) gives $\hat{\omega}_{1}=$ const. and since eigenfunctions can be multiplied by an arbitrary constant we can set $\hat{\omega}_{1}=1$ without loss of generality. Then (65) gives

$\hat{u}_{2}=\frac{\mathrm{i} \hat{k}_{x}}{2}\left(z^{2}-\frac{1}{4}\right)$

the constant of integration being determined by the requirement that $u_{z}=0$ at $z= \pm 1 / 2$. (67) gives

$\hat{\theta}_{0}=\mathrm{i} \hat{k}_{x}\left(a_{0}+a_{1} z+\frac{S z^{2}}{16 \hat{E}}\left(\frac{8-\hat{a}^{2}}{\hat{a}^{2}}\right)+\frac{S z^{4}}{24 \hat{E}}\right)$,

where again the boundary conditions on $\theta$ determine $a_{1}=0$ and

$a_{0}=\frac{S}{64 \hat{E}}\left(\frac{5}{6}-\frac{8}{\hat{a}^{2}}\right)$ 
This completes the solution of the leading order equations. At the next order, $O\left(\epsilon^{2}\right)$ for (16), we obtain

$\frac{\mathrm{d} \hat{\omega}_{2}}{\mathrm{~d} z}=-\hat{a}^{2} \hat{\theta}_{0}$,

which has solution

$\hat{\omega}_{2}=-\mathrm{i} \hat{k}_{x} \hat{a}^{2}\left(a_{0} z+\frac{S z^{3}}{48 \hat{E}}\left(\frac{8-\hat{a}^{2}}{\hat{a}^{2}}\right)+\frac{S z^{5}}{120 \hat{E}}\right)$,

since the constant of integration can be absorbed into the leading order constant. Equation (15) at $O\left(\epsilon^{3}\right)$ gives

$\hat{\sigma} \hat{\omega}_{1}+\mathrm{i} \hat{k}_{x} z \hat{\omega}_{2}-\mathrm{i} \hat{k}_{y} \hat{u}_{2}=\frac{\mathrm{d} \hat{u_{3}}}{\mathrm{~d} z}$.

Integrating this equation gives $\hat{u}_{3}$ as an odd polynomial of degree 7 . The boundary conditions require the constant of integration vanishes, but they also require that the odd polynomial vanishes at $z= \pm 1 / 2$. This condition gives the required formula for the growth rate, which after some manipulation can be written

$\hat{\sigma}=\frac{\hat{k}_{x} \hat{k}_{y}}{12}-\frac{17 \hat{k}_{x}^{2} \hat{a}^{2} S}{20160 \hat{E}}+\frac{\hat{k}_{x}^{2} S}{120 \hat{E}}$,

which in the unscaled units gives

$\sigma=\frac{k_{x} k_{y}}{12}-\frac{17 k_{x}^{2} a^{2} \operatorname{RiPr}}{20160 E}+\frac{k_{x}^{2} \operatorname{Pr}}{120 E}$.

The maximum growth rate occurs when

$\frac{\partial \sigma}{\partial k_{x}}=\frac{k_{y}}{12}+\frac{k_{x} P r}{60 E}-\frac{17 k_{x}^{3} \operatorname{RiPr}}{5040 E}-\frac{17 k_{x} k_{y}^{2} \operatorname{RiPr}}{10080 E}=0$,

$\frac{\partial \sigma}{\partial k_{y}}=\frac{k_{x}}{12}-\frac{17 k_{x}^{2} k_{y} R i P r}{10080 E}=0$.

Together these give

$k_{x}=\left(\frac{84}{17 R i}\right)^{1 / 2}, k_{y}=\left(\frac{8400}{17 R i}\right)^{1 / 2} \frac{E}{P r}$

and so the maximum growth is

$\sigma_{\text {nonax }}=\frac{35}{17 R i}\left(\frac{E}{P r}+\frac{P r}{100 E}\right)$.

We see that the $3 \mathrm{D}$ mode is always unstable in this regime, but its growth rate is very small, because $R i$ is large. If we compare these formulae with the results from the fully $3 \mathrm{D}$ numerical code shown in Fig. 3c, at $R i=2000, E=10^{-5}, \operatorname{Pr}=10^{-4}$ we obtain $k_{x}=k_{y}=0.0497$ and $\sigma_{\max }=0.00020588$. This compares very satisfactorily with the numerically obtained results, $k_{x}=k_{y}=$ $0.05, \sigma_{\max }=0.000206$.

We can now find the cross-over point $\operatorname{Pr}_{C x}$, given by numerical computation in Table 1 , by using the asymptotic formulae (58) and (79). The cross-over point is then the solution of

$$
\begin{array}{r}
E\left(1+\frac{4}{R o^{2}}\right)\left[\frac{2\left(0.25-\operatorname{RiPr}_{C x}\right)^{3 / 2} R o^{3}}{3^{3 / 2} E\left(1+R o^{2} / 4\right)^{3 / 2}}-\pi^{2}\right]= \\
\frac{35}{17 R i}\left(\frac{E}{P r_{C x}}+\frac{\operatorname{Pr}_{C x}}{100 E}\right) .
\end{array}
$$

With $E=10^{-5}, R i=2000$, Ro $=1$ we obtain $\operatorname{Pr}_{C x}=0.121805 \times 10^{-3}$, in excellent agreement with the Table 1 value.
Note that from Eq. (12),

$$
u_{y}=\frac{1}{k_{x}^{2}+k_{y}^{2}}\left(\mathrm{i} k_{y} \frac{\mathrm{d} u_{z}}{\mathrm{~d} z}-\mathrm{i} k_{x} \omega_{z}\right)
$$

From (61), (62) and (63) we see the term involving $\mathrm{d} u_{z} / \mathrm{d} z$ in (81) is negligible, and $u_{y}$ is $O\left(\omega_{z} / \epsilon\right)$. However, $u_{z}$ is $O\left(\epsilon \omega_{z}\right)$ from (63) and (62), so $u_{y}$ is $O(R i)$ larger than $u_{z}$. This means that the particle motions lie in the very narrow wedge bounded by $z=0$ with angle only $\left(k_{x}^{2}+k_{y}^{2}\right) / 8$. This 3D instability is therefore essentially the classical Eady baroclinic instability (see e.g. Drazin $\&$ Reid 1981), the only difference being that because of our very low Prandtl number, thermal diffusion is important. In the limit in which $E$ is so small that thermal diffusion in (17) can be ignored, we recover the classical Eady problem, which has instability with very slow growth rate with the wavenumbers $k_{x}$ and $k_{y}$ small at large $R i$.

In spherical geometry, azimuthal waves have dependence $\sim \exp \operatorname{im} \phi$, and $m=1$ is the smallest possible non-axisymmetric wavenumber. It is therefore relevant to consider the problem with $k_{x}$ small but fixed in (75). If we maximise $\sigma$ over $k_{y}$ only using (77), we obtain

$\sigma=\frac{35 E}{17 \operatorname{PrRi}}+\frac{k_{x}^{2} \operatorname{Pr}}{120 E}-\frac{17 \operatorname{RiPr} k_{x}^{4}}{20160 E}$.

This becomes stable as $k_{x}$ increases, and marginal stability is given by

$k_{x}^{2}=\frac{84}{17 R i}\left(1+\sqrt{1+\frac{100 E^{2}}{P r^{2}}}\right)$.

For $k_{x}^{2}$ greater than this value, the $3 \mathrm{D}$ mode is stable. In tachocline conditions, $E / P r$ is probably small, so $k_{x} \approx$ $2.22 R i^{-1 / 2}$.

\section{Conclusions}

The large vertical shear in the tachocline means that it is in a baroclinic state with a latitudinal temperature gradient, and is therefore subject to baroclinic instabilities. However, the very strong stabilising stratification in the slow tachocline means that such instabilities are much weakened. Nevertheless, in the slow tachocline region where we expect evolution to occur on timescales of hundreds of years, these instabilities will be very significant.

In tachocline conditions, where $R i$ is large and $P r$ and $E$ are small only two types of instability survive. The 3D mode, which is an Eady mode modified by the large thermal diffusivity, survives at large $R i$ but its growth rate becomes small, essentially $O(\Omega / R i)$, as does the horizontal wavenumber $k_{x}$. It is this last feature which is most problematic for Eady modes in the tachocline. From (83), if $k_{x}$ is fixed at a value larger than $\sim 2.3 R i^{-1 / 2}$, then the Eady mode is stabilised. The smallest non-axisymmetic wavenumber possible in spherical geometry is $m=1$. If the thickness of the tachocline is $d$, then the smallest allowed $k_{x}$ is $d / R_{t}$, where $R_{t}$ is the distance of the tachocline from the solar centre. The tachocline is believed to be thin, but it is uncertain that it is so thin that $d / R_{t}<2.3 R i^{-1 / 2}$, neccesary for these 3D Eady modes to be unstable. However, if this criterion is satisfied, the growth rate of the Eady modes is $\sim \Omega / R i$ which although slow, is fast enough in the slow tachocline for these modes to be physically relevant. Also for the zone affected by penetrative convection the Richardson number falls to order one 
values and here Eady modes could play a very significant role, as they do in the Earth's atmosphere.

Since non-axisymmetric baroclinic instabilities play such a dominant role in the Earth's weather system, it is worth considering the essential difference between the Earth's atmosphere and the slow tachocline. Both the Earth's atmosphere and the tachocline are stably stratified, but in the slow tachocline the Richardson number is much larger, i.e. the stabilising stratification is more dominant. Hence in the tachocline non-axisymmetric waves grow slowly and the vertical shear disrupts them before they grow significantly. In the Earth, lower $R i$ means they grow too fast for shear to disrupt them. However, although these non-axisymmetric modes are probably not important in the deeper regions of the tachocline, it would be premature to ignore them altogether. In the regions were penetrative convection is significant, they may play an important role in the dynamics.

By contrast, the axisymmetric Goldreich-Schubert type modes are probably important in the slow tachocline. As is apparent from (37), (53) and (58) these modes are unstable if $\operatorname{RiPr}<1 / 4$, and if $\operatorname{RiPr}$ is small, the growth rate of $\kappa k^{2} R i^{-1}$ can be as large as $O(\Omega)$ due to the short thermal diffusion time arising from the large wavenumber modes. This suggests that these modes may lead to efficient angular momentum transport in the tachocline in all regions where $\operatorname{RiPr}<1 / 4$. However, we need to consider how much angular momentum can be transported by these modes in view of their fingering nature. This can only be addressed by a fully nonlinear treatment. Also we have ignored the important effects of magnetic fields (see e.g. Acheson 1978; Menou et al. 2004), which may well be significant for these instabilities. Both these issues will be addressed in future work.
Finally, we note that rather unusually, this problem lends itself to asymptotic methods as well as numerical methods in both the linear and nonlinear regime. This makes it a particularly promising model for many aspects of tachocline research.

\section{References}

Acheson, D. J. 1978, Phil. Trans. Roy. Soc. Lond. A, 289, 459

Christensen-Dalsgaard, J., \& Schou, J. 1988, in Seismology of the Sun \& Sun-like Stars, ed. V. Domingo, \& E. J. Rolfe, ESA SP-286, 149

Christensen-Dalsgaard, J., \& Thompson, M. J. 2007, in Solar Tachocline, ed. D. W. Hughes, R. Rosner, \& N. O. Weiss (Cambridge University Press)

Drazin, P. G., \& Reid, W. H. 1981, Hydrodynamic stability (Cambridge University Press)

Fricke, K. J. 1968, Z. Astrophys., 68, 317

Goldreich, P., \& Schubert, G. 1967, ApJ, 150, 571

Gilman, P. A., \& Cally, P. S. 2007, in Solar Tachocline, ed. D. W. Hughes, R. Rosner, \& N. O. Weiss (Cambridge University Press), 243

Gough, D. O. 2007, in Solar Tachocline, ed. D. W. Hughes, R. Rosner, \& N. O. Weiss (Cambridge University Press), 1

Gough, D. O., \& McIntyre, M. E. 1998, Nature, 394, 755

Hughes, D. W. 2007, in Solar Tachocline, ed. D. W. Hughes, R. Rosner, \& N. O. Weiss (Cambridge University Press), 275

Knobloch, E., \& Spruit H. C. 1982, A\&A, 113, 261

Menou, K., Balbus, S. A., \& Spruit, H. C. 2004, ApJ, 607, 564

Miesch, M. S. 2005, Living Rev. Sol. Phys., 2, 1 www. livingreviews. org/lrsp-2005-1

Miller, T. L., \& Antar, B. N. 1986, J. Atmos. Sci., 43, 329

Spiegel, E. A., \& Zahn, J.-P. 1992, A\&A, 165, 106

Tobias, S. M. 2005, in Fluid Dynamics and Dynamos in Astrophysics and Geophysics, ed. A. M. Soward, et al. (Boca Raton: CRC), 193

Tobias, S. M., \& Weiss, N. O. 2007, in Solar Tachocline, ed. D. W. Hughes, R. Rosner, \& N. O. Weiss (Cambridge University Press), 319 